\title{
MATHEMATICAL LOGIC QUARTERLY
}

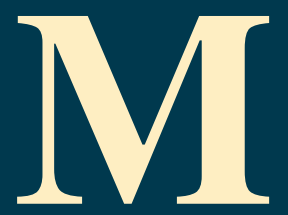

A Journal for Mathematical Logic, Foundations of Mathematics, and Logical Aspects of Theoretical Computer Science
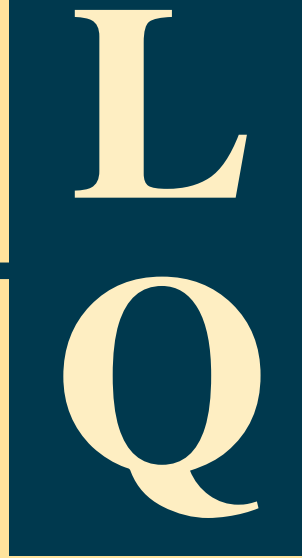

www.mlq-journal.org

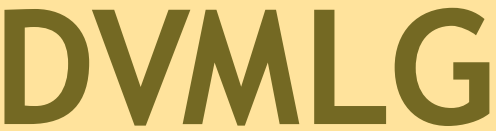

Published under the auspices of the

Deutsche Vereinigung für Mathematische Logik und für

Grundlagenforschung der Exakten Wissenschaften (DVMLG)

\section{Managing Editors}

Benedikt Löwe, Amsterdam \& Hamburg Klaus Meer, Cottbus

Pavel Pudlak, Prague

\section{Editorial Board}

Marat Arslanov, Kazan

John T. Baldwin, Chicago, IL

Douglas S. Bridges, Canterbury

Armin Hemmerling, Greifswald

Ramon Jansana, Barcelona

Carl G. Jockusch, Urbana, IL

Alexander Kechris, Pasadena, CA

Ulrich Kohlenbach, Darmstadt

\section{Editorial Assistant}

Peter van Ormondt, Amsterdam

\section{Honorary Editor}

Günter Asser, Greifswald

H. Dugald Macpherson, Leeds Johann Makowsky, Haifa Andrzej Rosłanowski, Omaha, NE Jörg Rothe, Düsseldorf

Wilfried Sieg, Pittsburgh, PA

Stephen G. Simpson, State College, PA W. Hugh Woodin, Berkeley, CA 


\title{
Cell decomposition and definable functions for weak $p$-adic structures
}

\author{
Eva Leenknegt* \\ Department of Mathematics, Purdue University, 150 N. University Street, West Lafayette, IN 47907-2067, \\ United States of America
}

Received 2 April 2012, revised 30 July 2012, accepted 13 August 2012

Published online 17 October 2012

Key words Cell decomposition, quantifier elimination, p-adic geometry, minimality. MSC (2010) 03C07, 03C10, 03C64, $11 \mathrm{U} 09$

\begin{abstract}
We develop a notion of cell decomposition suitable for studying weak $p$-adic structures (reducts of $p$-adic fields where addition and multiplication are not (everywhere) definable). As an example, we consider a structure with
\end{abstract} restricted addition.

(c) 2012 WILEY-VCH Verlag GmbH \& Co. KGaA, Weinheim

\section{Introduction and first definitions}

Results for real fields have always been a big source of inspiration for the study of $p$-adic fields. An example of this is the concept of o-minimality (cf., e.g., [16]) which inspired Haskell and Macpherson [5] to develop a similar concept, P-minimality, for $p$-adic fields. A difference between those concepts is that o-minimality also covers reducts of real closed fields $R$ (cf. $[11,13,14]$ ) while P-minimality focuses on expansions of the language of valued fields. A natural question is whether the concept of P-minimality can be expanded to include structures for which the corresponding language is weaker than the ring language.

A structure $(R, \mathcal{L})$ is o-minimal if the $\mathcal{L}$-definable subsets of $R$ coincide with the subsets definable in the language containing only the symbol ' $<$ '. In the $p$-adic context, one might consider the analogous relation $D^{(2)}(x, y) \leftrightarrow \operatorname{ord}(x)<$ ord $(y)$. A possible candidate-expansion for P-minimality would then be to study those structures $(K, \mathcal{L})$ for which the $\mathcal{L}$-definable subsets of $K$ coincide with the $\left(D^{(2)}\right)$-definable subsets; let us call such structures $D^{(2)}$-minimal. However, even very basic structures like $\left(\mathbb{Q}_{p} ; D^{(2)},+\right)$ are not $D^{(2)}$-minimal: a set $\left\{x \in \mathbb{Q}_{p} \mid\right.$ ord $(x+a)<$ ord $\left.(b)\right\}$ is in general not $\left(D^{(2)}\right)$-definable. Moreover, P-minimal structures are not $\left(D^{2}\right)$-minimal, as for example the sets of nonzero $n$-th powers $P_{n}$ are not $\left(D^{(2)}\right)$-definable. (Note that in this paper 'definable' will always mean 'definable with parameters'.)

To obtain a more interesting minimality concept, we shall need to require that the structure of $p$-adic semialgebraic sets satisfies our minimality property. We shall consider reducts $(K, \mathcal{L})$ of $\left(K, \mathcal{L}_{\text {ring }}\right)$, where $K$ is a $p$-adically closed field, and the $\mathcal{L}$-definable subsets of $K$ are exactly the $\mathcal{L}_{\text {ring-definable (semi-algebraic) subsets }}$ of $K$. A first step towards understanding such structures is to describe the boundaries of our 'playing field': identify the relations and functions that, as a bare minimum, would have to be definable in such a structure. In our paper [2], we concluded that all semi-algebraic subsets of $K$ can be defined using only the relations

$$
R_{n, m}(x, y, z) \leftrightarrow y-x \in z Q_{n, m} .
$$

The sets $Q_{n, m}$, which for $K=\mathbb{Q}_{p}$ can be defined as $\bigcup_{k \in \mathbb{Z}} p^{k n}\left(1+p^{m} \mathbb{Z}_{p}\right)$, are a variation on the sets of $n$-th powers $P_{n}$ that one encounters in the study of $p$-adic semi-algebraic sets. A more general definition will be given in Section 1.1. Write $\mathcal{L}_{M}$ for the language $\mathcal{L}_{M}:=\left(\left\{R_{n, m}\right\}_{n, m \in \mathbb{N}}\right)$. Expansions $(K, \mathcal{L})$ of $\left(K, \mathcal{L}_{M}\right)$ will be called $\mathcal{L}_{M}$-minimal if the $\mathcal{L}$-definable subsets of $K$ coincide with the $\mathcal{L}_{M}$-definable subsets of $K$.

*e-mail: eleenkne@ @math.purdue.edu 
Now that we know which structures we want to consider, the second step will be to describe their definable sets. Historically, cell decomposition has proved to be a very useful tool in studying definability questions. Examples include o-minimal cell decomposition in the real case, and Denef's cell decomposition for $p$-adic semi-algebraic sets, which can be stated as follows:

Theorem 1.1 (Denef, $[3,4]$ ) Let $K$ be a finite field extension of $\mathbb{Q}_{p}$. Any semi-algebraic set $X \subseteq K^{k+1}$ can be partitioned as a finite union of cells of the form

$$
\left\{(x, t) \in D \times K \mid \text { ord }\left(a_{1}(x)\right) \square_{1} \text { ord }(t-c(x)) \square_{2} \text { ord }\left(a_{2}(x)\right), t-c(x) \in \lambda P_{n}\right\},
$$

where $D$ is a semi-algebraic subset of $K^{k}$ and $c(x), a_{i}(x)$ are semi-algebraic functions. The symbols $\square_{i}$ denote ' $<$ ' or 'no condition'.

Among other applications, Denef used this result to give a new proof of Macintyre's quantifier elimination result [10]. The result was also important for Mourgues [12] result on P-minimal cell decomposition.

In [2], we showed that the relation $D^{(3)}(x, y, z) \leftrightarrow$ ord $(x-y)<$ ord $(z-y)$ is definable in the language $\mathcal{L}_{M}=\left(\left\{R_{n, m}\right\}_{n, m}\right)$. Because of this, cells $C \subset K$ are $\mathcal{L}_{M}$-definable, which is what we wanted. However, to get a language that is more convenient to work with, we shall replace $D^{(3)}$ by the slightly stronger relation $D^{(4)}$, defined as

$$
D^{(4)}(x, y, z, t) \leftrightarrow \operatorname{ord}(x-y)<\operatorname{ord}(z-t) .
$$

The resulting language $\mathcal{L}_{\text {dist }}=\left(D^{(4)},\left\{R_{n, m}\right\}_{n, m}\right)$ is strictly stronger than $\mathcal{L}_{M}$, as there are sets which are $\mathcal{L}_{\text {dist }}$-definable, but not $\mathcal{L}_{M}$-definable [8].

Our definition of cells is inspired by Denef's $p$-adic cells, but with some modifications, the first being that we use the sets $Q_{n, m}$ instead of the usual $P_{n}$. A second difference is that we shall only require the relation ord $\left(a_{i}(x)\right)<$ ord $(t)$ to be definable, and not necessarily the function $a_{i}(x)$ itself. This change is motivated by the observation that the function $(x, y) \mapsto x-y$ is not necessarily definable in all languages that contain a symbol for the relation $D^{(4)}$. We shall call this weak cell decomposition as opposed to (strong) cell decomposition results that only use definable functions.

While working on the cell decomposition results presented in this paper, we noticed that many of those results were valid for a much wider class of fields than just $p$-adically closed fields. In particular, we do not need to assume that the field is henselian, and our results will work in any characteristic. For this reason, even though our original motivation was the study of $p$-adically closed fields, we present our results for $\left(\mathbb{F}_{q}, \mathbb{Z}\right)$-fields: valued fields with residue field isomorphic to $\mathbb{F}_{q}$ and value group elementarily equivalent to $\mathbb{Z}$. So this paper is really about cell decomposition techniques for expansions of $\left(K, \mathcal{L}_{\text {dist }}\right)$, where $K$ is an $\left(\mathbb{F}_{q}, \mathbb{Z}\right)$-field.

Let us now give a brief overview of the contents of this paper. We first explain the ideas behind the sets $Q_{n, m}$ in Section 1.1, and then give a formal definition of our concept of cells in Section 1.2. In Section 2, we state some cell decomposition results valid for all expansions of $\mathcal{L}_{\text {dist }}$, and we show how these results can be used to study the language $\mathcal{L}_{\text {dist }}$ itself.

In our definition of cells, we made a distinction between weak and strong cell decomposition, depending on whether or not the functions used were definable. In Section 3 we investigate the definable functions of structures that admit weak cell decomposition. In particular, we shall focus on the existence of definable Skolem functions. The reason for this is a result by Mourgues for P-minimal fields, stating that a structure has (strong) cell decomposition if and only if it admits definable Skolem functions. When restricting our attention to $p$-adically closed fields, we can obtain a similar result for $\mathcal{L}_{M}$-minimal expansions of $\mathcal{L}_{\text {dist }}$. More precisely, if an $\mathcal{L}_{M}$-minimal expansion of $\left(K, \mathcal{L}_{\text {dist }}\right)$ has definable Skolem functions, then it admits strong cell decomposition. If we assume that addition and some scalar multiplication are definable, we also obtain the converse: a structure that has strong cell decomposition will automatically have definable Skolem functions as well.

However, this result is not as strong as it seems to be. In P-minimality, requiring the existence of definable Skolem functions is a relatively minor assumption, as there are no known examples of structures that do not have such functions. For the weaker structures we study, we get a different picture: $\left(K, \mathcal{L}_{\text {dist }}\right)$ itself is an example of a structure having no definable Skolem functions. So the really interesting questions are whether or not every expansion of $\left(K, \mathcal{L}_{\text {dist }}\right)$ admits weak cell decomposition, and under which conditions a structure would have definable Skolem functions. 
At this time, we cannot answer the first question, and we can only give a conjecture for the second question. It is known that every o-minimal expansion of the structure $(R,+,-,<, 0,1)$ has definable Skolem functions (here $R$ is a real closed field). Based on the structures we have studied, it seems that expansions of $\mathcal{L}_{\text {dist }}$ cannot have Skolem functions unless addition and sufficient scalar multiplication are definable. Section 4 provides an example of a structure where we have full scalar multiplication, and where addition is definable on large open sets, but which still does not admit definable Skolem functions.

The expansions considered in this paper are still very basic. Adding a symbol for either addition [7] or (restricted) multiplication [8] is also possible, but for this we refer to [7] and [8,9] respectively.

\subsection{Definition of the sets $Q_{n, m}$}

The field of $p$-adic numbers admits elimination of quantifiers in the language $\mathcal{L}_{\mathrm{Mac}}$, which is the ring language, extended with predicates $P_{n}$ for the sets of $n$-th powers. Note that by Hensel's lemma, there exists $r \in \mathbb{N}$ such that $1+p^{r} \mathbb{Z}_{p} \subset P_{n}$. Hence, elements $x \in \mathbb{Q}_{p}$ can be written as $x=\lambda p^{k n} u$, with $k \in \mathbb{Z}, u \in 1+p^{r} \mathbb{Z}_{p}$. From this, one can see that cosets $\lambda P_{n}$ encode certain information concerning the value group and the angular components of their elements. This is essentially the reason why $\mathbb{Q}_{p}$ admits elimination of quantifiers in $\mathcal{L}_{\text {Mac }}$.

However, this only works because $\mathbb{Q}_{p}$ and other $p$-adically closed fields are Henselian. Write $\mathcal{L}$ for the language of valued fields, and assume that $K$ is a general valued field. To obtain quantifier elimination for $\left(K, \mathcal{L}_{\mid}\right)$, Basarab and Kuhlmann [1,6] proposed to add symbols for additive-multiplicative congruences (amc-structures). Amc-structures are quotient groups $K^{\times} /(1+I)$, for ideals $I \subset R_{K}$ (where $R_{K}$ is the valuation ring of $K$ ). For example, if $I=M_{K}$, the maximal ideal of $R_{K}$, then the corresponding amc-structure encodes information about the value group and the angular component modulo $\pi$.

We shall use a variation on amc-structures, that encodes similar information as the sets $P_{n}$, even when Hensel's lemma does not hold. In particular, we consider the following class of fields.

Definition 1.2 Let $\mathbb{F}_{q}$ be the finite field with $q$ elements and $\mathbb{Z}$ the ordered abelian group of integers. We define an $\left(\mathbb{F}_{q}, \mathbb{Z}\right)$-field $K$ to be a valued field with residue field isomorphic to $\mathbb{F}_{q}$ and value group elementarily equivalent to $\mathbb{Z}$.

Write ord $: K \rightarrow \Gamma_{K}$ for the valuation on $K$, where $\Gamma_{K}$ denotes the value group of $K$. Let $\pi$ be a fixed element of smallest positive order. The valuation is normalized such that ord $(\pi)=1$. Write $M_{K}$ for the maximal ideal of the valuation ring $R_{K}$ of $K$.

A first class of examples of $\left(\mathbb{F}_{q}, \mathbb{Z}\right)$-fields are $p$-adically closed fields, that is: henselian $\left(\mathbb{F}_{q}, \mathbb{Z}\right)$-fields of characteristic zero, where $q=p^{r}$ and the dimension of $R_{K} / p R_{k}$ regarded as a vector space over $\mathbb{F}_{p}$ is finite. Note that in general, an $\left(\mathbb{F}_{q}, \mathbb{Z}\right)$-field need not be of characteristic zero: fields like $\mathbb{F}_{q}(t)$ and $\mathbb{F}_{q}((t))$ are also examples.

For each integer $n>0$, let $P_{n}$ be the set of nonzero $n$-th powers in $K$. For each $m>0$, we shall define sets $Q_{n, m}$ using angular component maps. The following lemma shows that such maps exist and that they can be defined in a unique way.

Lemma 1.3 For each integer $m>0$, there is a unique group homomorphism

$$
a c_{m}: K^{\times} \rightarrow\left(R_{K} \bmod \pi^{m}\right)^{\times}
$$

such that $a c_{m}(\pi)=1$ and such that $a c_{m}(u) \equiv u \bmod (\pi)^{m}$ for any unit $u \in R_{K}$.

Pro of. Cf. [2, Lemma 1.3].

Using these angular component maps, we can define sets $Q_{n, m}$, for any $m, n>0$, as follows:

$$
Q_{n, m}:=\left\{x \in P_{n} \cdot\left(1+M_{K}^{m}\right) \mid \operatorname{ac}_{m}(x)=1\right\} .
$$

For example if $K=\mathbb{Q}_{p}$, then $Q_{n, m}$ is just the set $\bigcup_{k \in \mathbb{Z}} p^{k n}\left(1+p^{m} \mathbb{Z}_{p}\right)$. Note that $Q_{n, m}$ is an open subgroup of finite index of $K^{\times}$(for the valuation topology). Moreover, $Q_{n, m}$ is definable in the language of valued fields $(+,-, \cdot, \mid)$. Indeed, in the proof of the above lemma it is observed that there exists $N \in \mathbb{N}$ such that ac ${ }_{m}(x)=1$ for all $x \in P_{N}$. Hence, the set consisting of all $x \in K$ for which ac ${ }_{m}(x)=1$ is just a finite union of cosets of $P_{N}$. 
For any element $\lambda \in K$, let $\lambda Q_{n, m}$ denote the set $\left\{\lambda t \mid t \in Q_{n, m}\right\}$. We shall sometimes use the alternative notation $\rho_{n, m}(x)=\lambda$ to express that $x \in \lambda Q_{n, m}$. The relation between $\rho_{n, m}(x+y), \rho_{n, m}(x)$ and $\rho_{n, m}(y)$ is investigated in the lemma below (the proof is left to the reader).

Lemma 1.4 Put $\delta \in\{-1,1\}$. Suppose that $\rho_{n, m}(a)=\lambda$ and $\rho_{n, m}(b)=\mu$, then

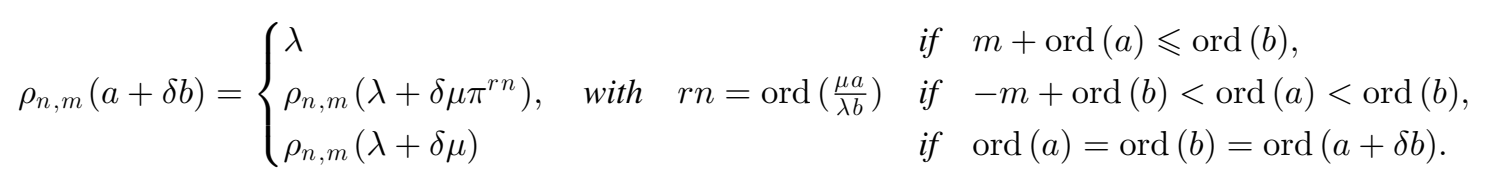

\subsection{Definition of $\left(\mathcal{L}, \Delta_{1}, \Delta_{2}\right)$-cells}

Let $K$ be an $\left(\mathbb{F}_{q}, \mathbb{Z}\right)$-field. As stated in the introduction, we shall be working with functions $f: K^{k} \rightarrow K$ for which the relation

$$
\operatorname{ord}(f(x))<\operatorname{ord}(t)
$$

is definable for all $(x, t) \in K^{k+1}$. We call these order-definable functions. Note that $f$ is not required to be a definable function. However, the following relations are always definable if the relation ord $(x)<\operatorname{ord}(y)$ is definable in our language:

Lemma 1.5 Let $\mathcal{L}$ be a language where the relation $D^{(2)}(x, y):=\operatorname{ord}(x)<\operatorname{ord}(y)$ is definable. Let $a_{1}(x), a_{2}(x)$ be two functions $K^{k} \rightarrow K$ that are order-definable in $\mathcal{L}$. The following relations are definable:

(1) $\operatorname{ord}(x)<\operatorname{ord}(y)+k$, for any $k \in \mathbb{Z}$;

(2) $\operatorname{ord}\left(a_{1}(x)\right) \square$ ord $(t)$;

(3) $\operatorname{ord}\left(a_{1}(x)\right) \square$ ord $\left(a_{2}(x)\right)+k$, for any $k \in \mathbb{Z}$;

where $\square$ may denote $<, \leqslant,=$, $\geqslant$ or $>$.

Proof. (1) For $k<0$, the relation ord $(x)+k>$ ord (y) can be expressed as

$$
\left(\exists u_{1}\right) \ldots\left(\exists u_{-k}\right)\left[\operatorname{ord}(x)>\operatorname{ord}\left(u_{1}\right)>\operatorname{ord}\left(u_{2}\right)>\cdots>\operatorname{ord}\left(u_{-k}\right)>\operatorname{ord}(y)\right] .
$$

For $k>0$, it is equivalent with

$$
\left(\forall u_{1}\right) \ldots\left(\forall u_{k}\right)\left[\left(\operatorname{ord}(x)<\operatorname{ord}\left(u_{1}\right)<\cdots<\operatorname{ord}\left(u_{k}\right)\right) \rightarrow \operatorname{ord}\left(u_{k}\right)>\operatorname{ord}(y)\right] .
$$

(2) For example if $\square$ denotes ' $=$ ', we can define the relation ord $\left(a_{1}(x)\right)=$ ord $(t)$ as

$$
\neg\left[\operatorname{ord}\left(a_{1}(x)\right)<\operatorname{ord}(t)\right] \wedge(\forall u)\left[\operatorname{ord}(t)<\operatorname{ord}(u) \rightarrow \operatorname{ord}\left(a_{1}(x)\right)<\operatorname{ord}(u)\right] .
$$

The other cases can be derived from this.

(3) The relation ord $\left(a_{1}(x)\right)<\operatorname{ord}\left(a_{2}(x)\right)$ is equivalent with

$$
(\exists t)\left[\operatorname{ord}\left(a_{1}(x)\right)<\operatorname{ord}(t) \wedge \operatorname{ord}\left(a_{2}(x)\right)=\operatorname{ord}(t)\right] .
$$

The rest can be derived from (1) and (2).

Let $\mathcal{L}$ be an expansion of $\left(D^{(2)},\left\{R_{n, m}\right\}_{n, m}\right)$. First we need some definitions.

Definition 1.6 A compatible set of functions is a tuple of sets $\left(\Delta_{1}, \Delta_{2}\right)$ satisfying the following conditions. For each $k>0$, let $\Delta_{1}^{(k)}$ be a collection of functions $K^{k} \rightarrow K$ that are order-definable in $(K, \mathcal{L})$. Let $\Delta_{2}^{(k)}$ be a collection of $\mathcal{L}$-definable functions $K^{k} \rightarrow K$, and put $\Delta_{i}=\bigcup_{k} \Delta_{i}^{(k)}$. For all $f_{1}, f_{2} \in \Delta_{2}^{(k)}$ we require that $f_{1}-f_{2} \in \Delta_{1}^{(k)}$.

Let $\left(\Delta_{1}, \Delta_{2}\right)$ be a compatible set of functions. We shall work with the following types of sets: 
Definition 1.7 An $\left(\mathcal{L}, \Delta_{1}, \Delta_{2}\right)$-precell in $K^{k}$ is a set $\left\{x \in K^{k} \mid \phi(x)\right\}$, where $\phi(x)$ is a Boolean combination of relations of the forms

$$
\operatorname{ord}\left(a_{1}(x)\right)<\operatorname{ord}\left(a_{2}(x)\right)+\ell, \quad \text { and } \quad b_{1}(x)-b_{2}(x) \in \lambda Q_{n, m},
$$

the $a_{i}(x)$ are functions in $\Delta_{1}^{(k)}, \ell \in \mathbb{Z}$ and the $b_{i}(x)$ are in $\Delta_{2}^{(k)}$.

Definition 1.8 An $\left(\mathcal{L}, \Delta_{1}, \Delta_{2}\right)$-cell $C_{c}^{D}\left(a_{1}, a_{2}, \lambda\right) \subseteq K^{k+1}$ is an $\mathcal{L}$-definable set of the following form:

$$
\left\{(x, t) \in D \times K \mid \text { ord }\left(a_{1}(x)\right) \square_{1} \text { ord }(t-c(x)) \square_{2} \text { ord }\left(a_{2}(x)\right), t-c(x) \in \lambda Q_{n, m}\right\},
$$

where $\lambda \in K, D$ is an $\left(\mathcal{L}, \Delta_{1}, \Delta_{2}\right)$-precell in $K^{k}, \square_{i}$ denotes ' $<$ ' or 'no condition', and the $a_{i}(x)$ are functions from $\Delta_{1}^{(k)}$. We call the function $c(x)$ the center of the cell and we require that $c(x) \in \Delta_{2}^{(k)}$.

When the language $\mathcal{L}$ and the set of functions $\left(\Delta_{1}, \Delta_{2}\right)$ are clear from the context, we shall sometimes just talk about precells and cells.

A structure $(K, \mathcal{L})$ then admits cell decompositon if there exist a compatible set of functions $\left(\Delta_{1}, \Delta_{2}\right)$ such that every definable set of $(K, \mathcal{L})$ can be partitioned as a finite union of $\left(\mathcal{L}, \Delta_{1}, \Delta_{2}\right)$-cells. The decomposition is said to be strong if every $f \in \Delta_{1}$ is $\mathcal{L}$-definable. Otherwise, the decomposition is said to be weak. The structures we study in this paper only allow weak cell decomposition. Cell decomposition for semi-algebraic sets and semilinear sets are (after a few straightforward adaptations) examples of strong cell decomposition.

\section{Cell Decomposition results}

Let $\mathcal{L}$ be a language expanding $\mathcal{L}_{\text {dist }}$. The relation $D^{(2)}$ is definable in all expansions of $\left(K, \mathcal{L}_{\text {dist }}\right)$, and hence our notion of cells makes sense for $(K, \mathcal{L})$. Assume that $\left(\Delta_{1}, \Delta_{2}\right)$ is a compatible set of functions. Then every Boolean combination of $\left(\mathcal{L}, \Delta_{1}, \Delta_{2}\right)$-cells can be partitioned into a finite number of $\left(\mathcal{L}, \Delta_{1}, \Delta_{2}\right)$-cells. This is an easy consequence of the following theorem.

Theorem 2.1 Let $\mathcal{L}$ be a language expanding $\mathcal{L}_{\text {dist }}$, and assume that $\left(\Delta_{1}, \Delta_{2}\right)$ is a compatible set of functions. Let $A_{1}, A_{2}$ be $\left(\mathcal{L}, \Delta_{1}, \Delta_{2}\right)$-cells with centers $c_{1}$, resp. $c_{2}$. The intersection $A_{1} \cap A_{2}$ can be written as a finite union of disjoint $\left(\mathcal{L}, \Delta_{1}, \Delta_{2}\right)$-cells $A$, each of which has a center which is a restriction of either $c_{1}$ or $c_{2}$.

Proof. By partitioning $C_{1}$ and $C_{2}$ further if necessary, we may suppose that they both use $Q_{n, m}$ with the same positive integers $m, n$, that is, that $C_{i}$ is of the form

$$
\left\{(x, t) \in D_{i} \times K \mid \operatorname{ord}\left(a_{1 i}(x)\right) \square_{1 i} \text { ord }\left(t-c_{i}\right) \square_{2 i} \text { ord }\left(a_{2 i}(x)\right), t-c_{i} \in \lambda_{i} Q_{n, m}\right\}
$$

for $i \in\{1,2\}$, where the symbols have their meaning as in Definition 1.8. Using Lemma 1.5, we can find a finite partitioning of $C_{1}$ in cells with the same center, such that on such a cell one of the following conditions holds for $k=1+m+n+\sum_{i, j=1,2}\left|k_{i j}\right|$ and some integer $\ell_{1}$ with $-k \leq \ell_{1} \leq k$.

$$
\begin{aligned}
\operatorname{ord}\left(t-c_{1}\right) & >\operatorname{ord}\left(c_{2}-c_{1}\right)+k, \\
\operatorname{ord}\left(t-c_{1}\right) & <\operatorname{ord}\left(c_{2}-c_{1}\right)-k, \\
\operatorname{ord}\left(t-c_{1}\right)+\ell_{1} & =\operatorname{ord}\left(c_{2}-c_{1}\right) .
\end{aligned}
$$

Hence, we may suppose that one of these conditions holds for $C_{1}$. Note that $(\mathrm{I})_{k}$ and (II) $)_{k}$ imply respectively

$$
\begin{aligned}
& \operatorname{ord}\left(t-c_{1}\right)>\operatorname{ord}\left(c_{2}-c_{1}\right)+k=\operatorname{ord}\left(t-c_{2}\right)+k, \\
& \operatorname{ord}\left(t-c_{2}\right)=\operatorname{ord}\left(t-c_{1}\right)<\operatorname{ord}\left(c_{1}-c_{2}\right)-k .
\end{aligned}
$$

If (I) $k$ holds on $C_{1}$, let $W=\left\{x \in D_{2} \mid\right.$ ord $\left(a_{12}(x)\right) \square_{12}$ ord $\left(c_{1}-c_{2}\right) \square_{22}$ ord $\left.\left(a_{22}(x)\right)\right\}$. Then one has, if $C_{1} \cap C_{2}$ is nonempty, that $C_{1} \cap C_{2}=(W \times K) \cap C_{1}$, which can easily be seen to be a finite disjoint union of $\left(\mathcal{L}, \Delta_{1}, \Delta_{2}\right)$-cells of the desired form. If (II) ${ }_{k}$ holds on $C_{1}$, then we may suppose, up to partitioning $C_{2}$, that (II) ${ }_{k}$ holds for all $(x, t) \in C_{1}$ and all $(x, t) \in C_{2}$. Also, we find that $\rho_{n, m}\left(t-c_{1}\right)=\rho_{n, m}\left(t-c_{2}\right)$, so either $C_{1} \cap C_{2}$ is empty or $C_{1} \cap C_{2}$ consists of all points $(x, t) \in\left(D_{1} \cap D_{2}\right) \times K$ satisfying the conditions

$$
\max _{i \in I}\left\{\operatorname{ord}\left(a_{1 i}(x)\right)\right\}<\operatorname{ord}\left(t-c_{1}\right)<\min _{i=1,2}\left\{\operatorname{ord}\left(a_{2 i}(x)\right)\right\},
$$


and

$$
t-c_{1} \in \lambda_{1} Q_{n, m}
$$

where $I$ consists of $i$ such that $\square_{1 i}$ is the condition $<$, and where the maximum over the empty set is $-\infty$. We know by Lemma 1.5 that relations of the form ord $\left(a_{i j}\right)<\operatorname{ord}\left(a_{l k}\right)$ are definable. It is then easy to see that $C_{1} \cap C_{2}$ can be partitioned into a finite number of disjoint $\left(\mathcal{L}, \Delta_{1}, \Delta_{2}\right)$-cells, which finishes the proof for this case.

We may suppose by symmetry (that is, up to reversing the role of $C_{1}$ and $C_{2}$ ) that, if (III) $\ell_{1}$ holds on $C_{1}$, then also

$$
\operatorname{ord}\left(t-c_{1}\right)+\ell_{1}=\operatorname{ord}\left(c_{2}-c_{1}\right)=\operatorname{ord}\left(t-c_{2}\right)+\ell_{2}
$$

holds with $\ell=\left(\ell_{1}, \ell_{2}\right)$ and $-k \leq \ell_{2} \leq k$. Suppose again that $C_{1} \cap C_{2}$ is nonempty. If one now fixes the residue classes of $c_{2}-c_{1}$ and of $t-c_{1}$ modulo $Q_{2 k n, 2 k n}$, then the conditions

$$
\text { ord }\left(c_{2}-c_{1}\right)=\operatorname{ord}\left(t-c_{2}\right)+\ell_{2} \text { and } t-c_{2} \in \lambda_{2} Q_{n, m}
$$

follow automatically from ord $\left(t-c_{1}\right)+\ell_{1}=$ ord $\left(c_{2}-c_{1}\right)$. (The exact relations are described in Lemma 1.4.) Hence, one can easily partition $C_{1} \cap C_{2}$ into finitely many $\left(\mathcal{L}, \Delta_{1}, \Delta_{2}\right)$-cells.

One of our main motivations for using cell decomposition is because it is a very useful tool for quantifier elimination. An example is Denef's proof of quantifier elimination for semi-algebraic sets [4]. The following lemma, which is closely inspired by this paper, will be used quite often.

Lemma 2.2 Let $\mathcal{L}$ be a language expanding $\left(D^{(2)},\left\{R_{n, m}\right\}_{n, m}\right)$, and assume that $\left(\Delta_{1}, \Delta_{2}\right)$ is a compatible set of functions. Let $C_{c}^{D}\left(a_{1}, a_{2}, \lambda\right) \subseteq K^{k+1}$ be an $\left(\mathcal{L}, \Delta_{1}, \Delta_{2}\right)$-cell. Suppose that for every $l \in \mathbb{N}$, the set $\left\{x \in K^{k} \mid \operatorname{ord}\left(a_{1}\right) \equiv l \bmod n\right\}$ can be partitioned as a finite union of $\left(\mathcal{L}, \Delta_{1}, \Delta_{2}\right)$-precells $\subseteq K^{k}$. Then the projection

$$
P:=\left\{x \in K^{k} \mid \exists t:(x, t) \in C_{c}^{D}\left(a_{1}, a_{2}, \lambda\right)\right\}
$$

can be partitioned in a finite number of $\left(\mathcal{L}, \Delta_{1}, \Delta_{2}\right)$-precells.

Proof. It is easy to check that $P$ consists of all $x \in D$ satisfying the following condition:

$$
P=\left\{x \in D \mid \exists \gamma \in \Gamma_{K}: \text { ord }\left(a_{1}(x)\right)<\gamma<\operatorname{ord}\left(a_{2}(x)\right), \gamma \equiv \operatorname{ord}(\lambda) \bmod n\right\} .
$$

Thus $P$ is the set of all $x \in D$ satisfying

$$
\exists \gamma \in \Gamma_{K}: \frac{\operatorname{ord}\left[a_{1}(x) \lambda^{-1}\right]}{n}<\gamma<\frac{\operatorname{ord}\left[a_{2}(x) \lambda^{-1}\right]}{n} .
$$

Now if ord $\left(a_{1}(x) \lambda^{-1}\right) \equiv \zeta \bmod n$, for $0 \leqslant \zeta<n$, then condition (1) is equivalent with ord $\left(a_{1}(x) \lambda^{-1}\right)+n-$ $\zeta<$ ord $\left(a_{2}(x) \lambda^{-1}\right)$, which can be simplified to

$$
\text { ord }\left(a_{1}(x)\right)+n-\zeta<\text { ord }\left(a_{2}(x)\right) \text {. }
$$

This completes the proof, since $D$ is a precell, (2) is a precell condition and by our assumption, the set $\{x \in$ $\left.K^{k} \mid \operatorname{ord}\left(a_{1}(x) \lambda^{-1}\right) \equiv \zeta \bmod n\right\}$ can be partitioned as a finite union of precells.

\subsection{Example: the language $\mathcal{L}_{\text {dist }}$}

We shall now use the results from the previous section to investigate the language $\mathcal{L}_{\text {dist }}$. More specifically, we shall show that any $\left(\mathbb{F}_{q}, \mathbb{Z}\right)$-field admits quantifier elimination in the language

$$
\mathcal{L}_{\text {dist }}^{\prime}:=\left(\left\{D_{k}^{(4)}\right\}_{k \in \mathbb{Z}},\left\{R_{n, m}\right\}_{n, m}\right),
$$

where

$$
D_{k}^{(4)}(x, y, z, t) \leftrightarrow \operatorname{ord}(x-y)<\operatorname{ord}(z-t)+k
$$


Definition 2.3 We call a polynomial $f(x) \in K\left[x_{1}, \ldots, x_{k}\right]$ an $\mathcal{L}_{\text {dist }}$-polynomial in variables $\left\{x_{1}, \ldots x_{k}\right\}$ if $f(x)$ has one of the following forms

$$
f(x)=a, \quad \text { or } \quad f(x)=\pi^{k}\left(x_{i}-a\right), \quad \text { or } \quad f(x)=\pi^{k}\left(x_{i}-x_{j}\right),
$$

where $a \in K, k \in \mathbb{Z} ; 1 \leqslant i, j \leqslant k$.

An $\mathcal{L}_{\text {dist }}$-cell will be an $\left(\mathcal{L}_{\text {dist }}, \Delta_{1}, \Delta_{2}\right)$-cell with the following specifications.

Definition 2.4 Let $\Delta_{\text {dist, } 1}^{(k)}$ be the set of all $\mathcal{L}_{\text {dist }}$-polynomials in $k$ variables. The set $\Delta_{\text {dist, } 2}^{(k)}$ consists of all constant functions $K^{k} \rightarrow K: x \mapsto a$, and projection maps $K^{k} \rightarrow K: x \mapsto x_{i}$ onto the coordinate functions. Put $\Delta_{\text {dist }}^{i}:=\bigcup_{k \geqslant 0} \Delta_{\text {dist }, i}^{(k)}$. An $\mathcal{L}_{\text {dist }}$-cell $\subseteq K^{k+1}$ is then an $\left(\mathcal{L}_{\text {dist }}, \Delta_{\text {dist }}^{1}, \Delta_{\text {dist }}^{2}\right)$-cell $\subseteq K^{k+1}$.

It is an easy consequence of Lemma 1.5 that $\mathcal{L}_{\text {dist }}$ polynomials are order-definable functions, so $\left(\Delta_{\text {dist }}^{1}, \Delta_{\text {dist }}^{2}\right)$ is clearly a compatible set of functions. Therefore the following holds for $\mathcal{L}_{\text {dist }}$-cells.

Proposition 2.5 Let $A_{1}, A_{2}$ be $\mathcal{L}_{\text {dist }}$ cells with centers $c_{1}$, resp. $c_{2}$. The intersection $A_{1} \cap A_{2}$ can be written as a finite union of disjoint $\mathcal{L}_{\text {dist }}$-cells $A$ with as center a restriction of either $c_{1}$ or $c_{2}$.

Proof. This follows from Theorem 2.1.

Proposition 2.6 Every $\left(\mathbb{F}_{q}, \mathbb{Z}\right)$-field admits elimination of quantifiers in the language $\mathcal{L}_{\text {dist }}^{\prime}$.

Pro of. It is clear that any $\mathcal{L}_{\text {dist }}$-cell (and any $\mathcal{L}_{\text {dist }}$-precell) is quantifier free definable in $\mathcal{L}_{\text {dist }}^{\prime}$. Moreover, for any $\mathcal{L}_{\text {dist }}$-polynomial $f(x)$, the relation ord $(f(x)) \equiv l \bmod n$ can be partitioned in a finite number of precells. (Indeed, this relation can be written as a finite disjunction of relations $x_{i}-a \in \lambda Q_{n, m}$ or $x_{i}-x_{j} \in \lambda Q_{n, m}$. )

Since the requirements of Lemma 2.2 are satisfied, it is now sufficient to show that any set that is quantifier free definable in $\mathcal{L}_{\text {dist }}^{\prime}$ can be partitioned as a finite union of $\mathcal{L}_{\text {dist }}$-cells.

By Proposition 2.5, we only need to check that the sets (and complements of these sets)

$$
\left\{x \in K^{k} \mid D_{r}^{(4)}\left(g_{1}, g_{2}, g_{3}, g_{4}\right)\right\} \quad \text { and } \quad\left\{x \in K^{k} \mid R_{n, m}\left(g_{1}, g_{2}, g_{3}\right)\right\},
$$

with $g_{i}(x) \in\left\{x_{1}, \ldots, x_{k}\right\} \cup K$ and $r \in \mathbb{Z}$ can be partitioned as a finite union of $\mathcal{L}_{\text {dist }}$-cells.

The fact that $R_{n, m}(x, y, z)$ is equivalent to

$$
\bigvee_{\lambda \in \Lambda_{n, m}} R_{n, m}(x, y, \lambda) \wedge R_{n, m}(0, z, \lambda)
$$

implies that $\left\{x \in K^{3} \mid R_{n, m}(x, y, z)\right\}$ can be written as a union of $\mathcal{L}_{\text {dist }}$-cells, by Theorem 2.1. The complement of this set can also be written as a union of disjoint $\mathcal{L}_{\text {dist }}$-cells, since $\left\{(x, y) \in K^{2} \mid \neg R_{n, m}(x, y, \lambda)\right\}$ can be written as a finite union of (disjoint) sets of the form $\left\{(x, y) \in K^{2} \mid R_{n, m}(x, y, \mu)\right\}$.

To complete the proof it suffices to check that the set

$$
A:=\left\{(x, t) \in K^{k+1} \mid \operatorname{ord}\left(t-c_{1}\right)<\operatorname{ord}\left(\pi^{n}\left(t-c_{2}\right)\right)\right\},
$$

with $c_{1}, c_{2} \in\left\{x_{1}, \ldots, x_{k}\right\} \cup K$, can be partitioned as a finite union of cells. We may suppose that $c_{1} \neq c_{2}$. Partition $K^{k+1}$ in the following way:

$$
\begin{aligned}
K^{k+1}= & \left\{(x, t) \in K^{k+1} \mid \operatorname{ord}\left(t-c_{1}\right)>\operatorname{ord}\left(c_{1}-c_{2}\right)\right\} \\
& \cup\left\{(x, t) \in K^{k+1} \mid \operatorname{ord}\left(t-c_{1}\right)<\operatorname{ord}\left(c_{1}-c_{2}\right)\right\} \\
& \cup\left\{(x, t) \in K^{k+1} \mid \operatorname{ord}\left(t-c_{1}\right)=\operatorname{ord}\left(c_{1}-c_{2}\right)\right\} .
\end{aligned}
$$

Since $A=A \cap K^{k+1}$, we can write $A$ as a union of sets on which one of the conditions in (3) holds. For example, on

$$
B=A \cap\left\{(x, t) \in K^{k+1} \mid \operatorname{ord}\left(t-c_{1}\right)>\operatorname{ord}\left(c_{1}-c_{2}\right)\right\},
$$

we have that ord $\left(t-c_{2}\right)=\operatorname{ord}\left(c_{1}-c_{2}\right)$, and therefore $B$ is equal to the set

$$
B=\left\{(x, t) \in K^{k+1} \mid \operatorname{ord}\left(c_{1}-c_{2}\right)<\operatorname{ord}\left(t-c_{1}\right)<\operatorname{ord}\left(\pi^{n}\left(c_{1}-c_{2}\right)\right)\right\} .
$$

It is clear that $B$ can be partitoned as a finite number of $\mathcal{L}_{\text {dist }}$-cells. The other cases are similar. 
Note our strategy: For the given language $\mathcal{L}$, we first try to find a suitable compatible set $\left(\Delta_{1}, \Delta_{2}\right)$, such that we get a system of $\left(\mathcal{L}, \Delta_{1}, \Delta_{2}\right)$-cells. Generally, we shall try to make sure that $\Delta_{2}$ contains only quantifier-free definable functions. To obtain a definitional expansion that has quantifier elimination, we shall then add symbols to $\mathcal{L}$ such that for each $f \in \Delta_{1}$, the relations

$$
\operatorname{ord}(f(x))<\operatorname{ord}(t)+k \text { and } \operatorname{ord}(f(x)) \equiv l \bmod n
$$

are quantifier free definable in the extended language $\mathcal{L}^{\prime}$. To obtain quantifier elimination for $\mathcal{L}^{\prime}$, it is then sufficient to show that quantifier free $\mathcal{L}^{\prime}$-definable sets can be partitioned as a finite number of $\left(\mathcal{L}, \Delta_{1}, \Delta_{2}\right)$-cells. (Unfortunately, this last step may require quite a lot of work.)

\section{Cell decomposition and definable (Skolem) functions}

\subsection{Definable functions}

The example of $\mathcal{L}_{\text {dist }}$-definable sets illustrates how we can use cell decomposition to obtain quantifier elimination results for a structure $(K, \mathcal{L})$. Cell decomposition results also provide a lot of information concerning the definable functions of a given structure. For instance, all $\mathcal{L}_{\text {dist }}$-definable functions must have the following form (and thus these structures have only trivial definable functions):

Lemma 3.1 Let $f: A \subseteq K^{k} \rightarrow K^{\ell}$ be an $\mathcal{L}_{\text {dist }}$-definable function. There exists a finite partition of $A$ in $\mathcal{L}_{\text {dist }}$-cells such that on each cell $C$ the function $f$ has the form

$$
f_{\mid C}: C \rightarrow K^{\ell}: x \mapsto\left(f_{1}(x), f_{2}(x), \ldots, f_{\ell}(x)\right),
$$

where $f_{i}(x)$ is either one of the variables $\left\{x_{1}, \ldots, x_{k}\right\}$ or a constant from $K$.

This is a direct consequence of the following more general observation:

Proposition 3.2 Assume that $(K, \mathcal{L})$ admits cell decomposition using $\left(\mathcal{L}, \Delta_{1}, \Delta_{2}\right)$-cells. Let $f: A \subseteq K^{k} \rightarrow$ $K^{\ell}$ be an $\mathcal{L}$-definable function. There exists a finite partion of $A$, such that on each cell $C$ in the decomposition there are functions $f_{i} \in \Delta_{2}^{(k)}$ such that $f_{\mid C}(x)=\left(f_{1}(x), f_{2}(x), \ldots, f_{\ell}(x)\right)$ for all $x \in C$.

Proof. First we note that $f$ can be written as

$$
f: A \subseteq K^{k} \rightarrow K^{\ell}: x \mapsto\left(f_{1}(x), \ldots, f_{\ell}(x)\right),
$$

where the coordinate functions $f_{i}: K^{m} \rightarrow K$ are all $\mathcal{L}$-definable functions. Therefore it suffices to prove the lemma for the case $\ell=1$. A function $f: A \subseteq K^{k} \rightarrow K$ is $\mathcal{L}$-definable if and only if

$$
\text { Graph } f=\{(x, t) \in A \times K \mid t=f(x)\}
$$

is an $\mathcal{L}$-definable set. This means there exists a finite partition of Graph $f$ in $\left(\mathcal{L}, \Delta_{1}, \Delta_{2}\right)$-cells $G$ of the form

$$
\left\{(x, t) \in D \times K \mid \text { ord }\left(a_{1}(x)\right) \square_{1} \text { ord }(t-c(x)) \square_{2} \text { ord }\left(a_{2}(x)\right), t-c(x) \in \lambda Q_{n, m}\right\},
$$

with $c(x) \in \Delta_{2}^{(k)}$. Since $f$ is a function, for each $x \in D$ there must be a unique $t$ such that $(x, t) \in G$. This uniqueness condition implies that $\lambda=0$, and thus $G$ must have the form $\{(x, t) \in D \times K \mid t=c(x)\}$.

\subsection{Cell decomposition and Skolem functions}

When studying definable functions, another natural question to ask is whether a language $\mathcal{L}$ has definable Skolem functions: for a given definable function $f: X \rightarrow Y$, does there exist a definable function $g: f(X) \rightarrow X$ such that $f \circ g=\operatorname{Id}_{f(X)}$ ?

In the P-minimal context, Mourgues showed that a P-minimal structure $(K, \mathcal{L})$ has definable Skolem functions if and only if the structure allows cell decomposition, using a notion of cells similar to what we called 'strong cells', i.e., using cells that are defined using only definable functions. We obtain similar results for $\mathcal{L}_{M}$-minimal extensions of $\left(K, \mathcal{L}_{\text {dist }}\right)$. 
Theorem 3.3 Let $K$ be a p-adically closed field, and suppose that $\mathcal{L} \supseteq \mathcal{L}_{\text {dist }}$. If an $\mathcal{L}_{M}$-minimal structure $(K, \mathcal{L})$ has definable Skolem functions, then it admits strong cell decomposition.

Pr o o f. Assume that $(K, \mathcal{L})$ has definable Skolem functions. This implies that, if $(K, \mathcal{L})$ has cell decomposition, say using $\left(\mathcal{L}, \Delta_{1}, \Delta_{2}\right)$-cells, then it admits strong cell decomposition. Indeed, let $f: K^{k} \rightarrow K$ be a function in $\Delta_{1}$. Since $f$ is order-definable in $\mathcal{L}$, the following set is $\mathcal{L}$-definable:

$$
A:=\left\{(x, t) \in K^{k+1} \mid \text { ord }(f(x))=\text { ord }(t)\right\} .
$$

Consider the projection map $\pi_{x}: A \rightarrow K^{k}:(x, t) \mapsto x$. Since $(\mathcal{L}, K)$ has definable Skolem functions, there exists a definable function $g: \operatorname{Im} \pi_{x} \rightarrow A: x \mapsto(x, a(x))$ such that $\left(\pi_{x} \circ f\right)(x)=x$ for all $x \in \operatorname{Im} \pi_{x}$. But then $a(x)$ is a definable function, such that for each $x \in K^{k}$, ord $(f(x))=\operatorname{ord}(a(x))$.

Let us now check that under our assumptions, $(K, \mathcal{L})$ does indeed admit cell decomposition. The proof is essentially the same as the original proof (for P-minimal structures) by Mourgues. For this reason, we shall only give a brief sketch, and refer the reader to [12] for details. Let $\mathcal{L}$ be an extension of $\mathcal{L}_{\text {dist }}$, and $S^{\prime} \subseteq K^{n+1}$ an $\mathcal{L}$-definable set, defined by a formula $\phi(y, x)$. As in [12], using a compactness argument, it can be shown that there exists a quantifier-free $\mathcal{L}_{\text {dist }}$-formula $\psi(z, x)$ such that

$$
K \models \forall y \exists z \forall x(\phi(y, x) \Leftrightarrow \psi(z, x)) .
$$

Write $\pi_{n}: K^{n+1} \rightarrow K^{n}$ for the projection onto the first $n$ coordinates. As in Lemma 3.3 of [12], it can follows from (4) that, if $(K, \mathcal{L})$ has definable Skolem functions, there exists $m$, an $\mathcal{L}_{\text {dist }}$-definable subset $S$ of $K^{m+1}$ and an $\mathcal{L}$-definable function $f: \pi_{n}\left(S^{\prime}\right) \rightarrow K^{m}$ such that for any $y \in \pi_{n}\left(S^{\prime}\right)$,

$$
\left\{x \in K \mid(y, x) \in S^{\prime}\right\}=\{x \in K \mid(f(y), x) \in S\} .
$$

Now use the same reasoning as in the proof of Theorem 3.5 of [12], reducing to $\mathcal{L}_{\text {dist }}$-cell decomposition instead of semi-algebraic cell decomposition. We need to assume that $K$ is a $p$-adically closed field, to ensure that the $\mathcal{L}_{\text {dist }}$-definable subsets of $K$ coincide with the $\mathcal{L}_{\text {ring }}$-definable subsets of $K$. (Only for such fields, $\mathcal{L}_{M}$-minimality is truly an expansion of the notion of P-minimality.)

In fact, we can obtain a stronger result: any structure that satisfies the conditions of Theorem 3.3 and has definable Skolem functions, has strong cell decomposition using continuous functions. The reason is that if $K$ is $p$-adically closed, then every $\mathcal{L}_{M}$-minimal structure $(K, \mathcal{L})$ is a reduct of the P-minimal structure $\left(K, \mathcal{L}_{\text {ring }}\right)$. This makes it possible to transfer some results about functions in P-minimal structures to the $\mathcal{L}_{M}$-minimal context. For example, we have that

Lemma 3.4 Let $K$ be a p-adically closed field and $(K, \mathcal{L})$ an $\mathcal{L}_{M}$-minimal structure.

(1) Let $f: K \rightarrow K$ be an $\mathcal{L}$-definable partial function. Then there is an open subset $U$ of $\operatorname{dom}(f)$ such that $\operatorname{dom}(f)-U$ is finite and $f_{\mid U}$ is continuous.

(2) Let $n>0$ and $g: K^{n} \rightarrow K$ be an $\mathcal{L}_{M}$-definable partial function, and let $X=\operatorname{dom}(f)$. Let $Y=$ $\{y \in X: g$ is defined and continuous in a neighbourhood of $y\}$. Then the topological dimension of $X \backslash Y$ is strictly smaller than $n$.

(The topological dimension of a set $S \subseteq K^{n}$ is the greatest integer $k \leqslant n$ for which there is a projection Proj : $K^{n} \rightarrow K^{k}$ such that $\operatorname{Proj}(S)$ has non-empty interior in $K^{k}$.)

Proof. The results stated in the lemma where originally proven by Haskell and MacPherson [5] in the P-minimal context. Note that any $\mathcal{L}_{M}$-minimal structure $(K, \mathcal{L})$ is either P-minimal (in which case the lemma holds), or $(K, \mathcal{L})$ is a reduct of the P-minimal structure of semi-algebraic sets $\left(K, \mathcal{L}_{\text {ring }}\right)$. In the latter case, the $\mathcal{L}$-definable function $f: \operatorname{dom}(f) \rightarrow K$ is also a semi-algebraic function in $\left(K, \mathcal{L}_{\text {ring }}\right)$. Applying the P-minimal version of the lemma, we find a semi-algebraic set $U$ such that $\operatorname{dom}(f)-U$ is finite and $f_{\mid U}$ is continuous. Since $\operatorname{dom}(f)$ is an $\mathcal{L}$-definable set and $\operatorname{dom}(f)-U$ is finite, the set $U$ must also be $\mathcal{L}$-definable, and hence this part of the lemma transfers to $\mathcal{L}_{M}$-minimal structures.

The second part can be checked similarly. ( $Y$ is $\mathcal{L}$-definable since the relation ord $(x-y)<$ ord $(x-z)$ is $\mathcal{L}_{M}$-definable.) 
Because these properties hold for $\mathcal{L}_{M}$-minimal structures, Theorem 3.3 can be strengthened:

Lemma 3.5 Let $K$ is a p-adically closed field. If an $\mathcal{L}_{M}$-minimal expansion $(K, \mathcal{L})$ of $\left(K, \mathcal{L}_{\text {dist }}\right)$ admits strong cell decomposition using $\left(\mathcal{L}, \Delta_{1}, \Delta_{2}\right)$-cells, then all functions in $\left(\Delta_{1}, \Delta_{2}\right)$ can be assumed to be continuous.

Proof. The proof is the same as the original proof of Mourgues in [12], using Lemma 3.4 instead of the original P-minimal version.

If we omit the condition that $K$ has to be $p$-adically closed, we obtain a weaker version of Theorem 3.3: we can no longer be assured that definable Skolem functions imply the existence of cell decomposition, but if such a decomposition exists, it will be a strong decomposition.

How about the other direction? Does the fact that a structure admits strong cell decomposition automatically imply that it also has Skolem functions (as in the p-minimal case)? The answer is yes, under the additional assumptions that addition and a sufficient amount of scalar multiplication are definable.

How much scalar multiplication do we need exactly? For every coset $\lambda Q_{n, m}$, there should exist a representative $\lambda$ for which the function $x \mapsto \lambda x$ is definable. Write $\mathbb{P}_{K}$ for the prime field of $K$. Choose an element $\pi$ of $R_{K}$ of minimal positive valuation (hence, ord $(\pi)=1$ ). If $\mathbb{F}_{p}\left[\overline{a_{1}}, \ldots, \overline{a_{d}}\right]$ is the residue field of $K$, choose elements $a_{i} \in R_{K}^{\times}$such that $a_{i} \equiv \overline{a_{i}} \bmod \pi$. Then put $\mathbb{K}_{K}:=\mathbb{P}_{K}\left[\pi_{k}, a_{1}, \ldots, a_{d}\right]$. It is easy to see that this field contains an element of every coset of $\mathbb{Q}_{n, m}$ : if we put

$$
\Lambda_{n, m}:=\left\{c_{0}+c_{1} \pi+\ldots+c_{m-1} \pi^{m-1} \mid c_{i} \in\left\{a_{1}, \ldots, a_{d}\right\} \cup\{0\} \text { and } c_{0} \neq 0\right\},
$$

then clearly $\bigcup_{\lambda \in \Lambda_{n, m}} \lambda Q_{n, m}=K^{\times}$. If $K$ is a $p$-adically closed field, it is sufficient to require that scalar multiplication by elements of $\overline{\mathbb{Q}}^{K}$ (the algebraic closure of $\mathbb{Q}$ in $K$ ) is definable. Indeed, this subfield, which contains all elements of $K$ that are algebraic over $\mathbb{Q}$, has the following additional properties (as usual, we write ord for the valuation on $K$ ):

Lemma 3.6 If $K$ is a p-adically closed field, then $\overline{\mathbb{Q}}^{K}$ has the same residue field as $K$. Moreover, there exists $\pi \in \overline{\mathbb{Q}}^{K}$ such that ord $(\pi)=1$.

Proof. It is easy to see that $\overline{\mathbb{Q}}^{K}$ has the same residue field as $K$, since each $x \in \mathbb{F}_{K}$ is a simple root of $X^{q_{K}}-X$. The claim then follows by Hensel's Lemma. It is shown in Lemma 3.5ii of [15] that if $K$ is a $p$ adically closed field, and $F \subset K$ is algebraically closed in $K$, then $F$ contains an element $\pi$ of minimal positive valuation. Hence, $\overline{\mathbb{Q}}^{K}$ contains an element $\pi$ with ord $(\pi)=1$.

Proposition 3.7 Let $K$ be a p-adically closed field. Suppose that $\mathcal{L} \supseteq \mathcal{L}_{\text {dist }} \cup\{+\}$, and that multiplication by constants from $\overline{\mathbb{Q}}^{K}$ is definable in the structure $(K, \mathcal{L})$. If an $\mathcal{L}_{M}$-minimal structure $(K, \mathcal{L})$ admits strong cell decomposition, then it also has definable Skolem functions.

Pro o f. Assume that $(K, \mathcal{L})$ admits cell decomposition using $\left(\mathcal{L}, \Delta_{1}, \Delta_{2}\right)$-cells, and that every function in $\Delta_{1}$ is $\mathcal{L}$-definable. If suffices to check that given an $\left(\mathcal{L}, \Delta_{1}, \Delta_{2}\right)$-cell $C$ and the projection map $\pi_{x}: C \subset K^{l+1} \rightarrow$ $K^{\ell}$, there exists a definable function $g: \pi_{x}(C) \rightarrow C$ such that $\pi_{x} \circ g=\operatorname{Id}_{\pi_{x}(C)}$.

If the cell $C$ has a center $c(x) \neq 0$, we first apply a translation

$$
C \rightarrow C^{\prime}:(x, t) \mapsto(x, t-c(x)),
$$

to a cell $C^{\prime}$ with center $c^{\prime}(x)=0$. Since this translation is bijective, it is invertible. Therefore the problem is reduced to the following. Let $C$ be a cell of the form

$$
C=\left\{(x, t) \in D \times K \mid \text { ord }(b(x)) \square_{1} \text { ord }(t) \square_{2} \text { ord }(a(x)), t \in \lambda Q_{n, m}\right\},
$$

where $a(x), b(x)$ are $\mathcal{L}$-definable functions and $D$ is an $\left(\mathcal{L}, \Delta_{1}, \Delta_{2}\right)$-precell. We must show that there exists a definable function $g: \pi_{x}(C) \rightarrow C$ such that $\pi_{x} \circ g=\operatorname{Id}_{\pi_{x}(C)}$.

Given $x \in \pi_{x}(C) \subseteq D$, we have to find $t(x)$ such that $(x, t(x))$ satisfies the conditions

$$
\begin{aligned}
& \text { ord }(b(x)) \square_{1} \text { ord }(t(x)) \square_{2} \text { ord }(a(x)) \\
& t(x) \in \lambda Q_{n, m}
\end{aligned}
$$


We shall assume that each $\lambda \in \Lambda_{n, m}$. Then each representative has an order between 0 and $n-1$. Because of Lemma 3.6, we can assume that $\Lambda_{n, m} \subset \bar{Q}^{K}$, and hence multiplication and division by elements of $\Lambda_{n, m}$ is definable.

If $\lambda=0$, put $g(x)=(x, 0)$. From now on we assume that $\lambda \neq 0$.

If $\square_{1}=\square_{2}=$ 'no condition', we can simply put $g(x)=(x, \lambda)$.

If $\square_{2}=<$, we can define $g$ as follows. First partition $\pi_{x}(C)$ in parts $D_{\mu}$, such that

$$
D_{\mu}=\left\{x \in \pi_{x}(C) \mid a(x) \in \mu Q_{n, m}\right\} \text {. }
$$

(Note: if $\mu=0$, we can reduce to the cases were $\square_{2}=$ 'no condition'.) Our strategy is based on the fact that for every $x \in D$, there exists $k \in \mathbb{Z}$ such that $k$ satisfies

$$
\text { ord }\left(b_{1}(x)\right) \square_{1} \text { ord }(\lambda)+k n<\operatorname{ord}(a(x)) .
$$

Restricting to a set $D_{\mu}$, we construct an element $t(x)$ with order as close as possible to ord $(a(x))$. This ensures that $t(x)$ satisfies (5). The definition of $g$ on $D_{\mu}$ will depend on the respective orders of $\lambda$ and $\mu$.

If ord $(\lambda)<$ ord $(\mu)$, we can define $g_{\mid D_{\mu}}$ as $g_{\mid D_{\mu}}: D_{\mu} \rightarrow C: x \mapsto\left(x, \frac{\lambda}{\mu} a(x)\right)$. This means that we put $t(x)=\frac{\lambda}{\mu} a(x)$. Clearly $t(x) \in \lambda Q_{n, m}$. Also, since $-n<$ ord $\left(\frac{\lambda}{\mu}\right)<0$, we have that $0<$ ord $\left(\frac{a(x)}{t(x)}\right)<n$, and thus condition (5) must be satisfied.

If ord $(\lambda) \geqslant \operatorname{ord}(\mu)$, put $g_{D_{\mu}}: D_{\mu} \rightarrow C: x \mapsto\left(x, \frac{\lambda}{\pi^{n} \mu} a(x)\right)$.

If $\square_{1}=<$ and $\square_{2}=$ 'no condition', we choose $t(x)$ with order as close as possible to ord $(b(x))$. More specifically, if ord $(\lambda) \leqslant$ ord $(\mu)$, define $g$ as $g_{D_{\mu}}: D_{\mu} \rightarrow C: x \mapsto\left(x, \frac{\lambda \pi^{n}}{\mu} b(x)\right)$, and if ord $(\lambda)>\operatorname{ord}(\mu)$, put $g_{D_{\mu}}: D_{\mu} \rightarrow C: x \mapsto\left(x, \frac{\lambda}{\mu} b(x)\right)$.

The condition that multiplication by constants from $\mathbb{K}_{K}$ should be definable in $(K, \mathcal{L})$ is needed: for example the structure $\left(K ;+,-, D^{(4)},\left\{R_{n, m}\right\}_{n, m}\right)$ does not have definable Skolem functions for most $\left(\mathbb{F}_{q}, \mathbb{Z}\right)$-fields because we cannot define multiplication by enough scalars (in this structure, scalar multiplication is only definable for elements of $\mathbb{P}_{K}$ ). The examples in the next section support the conjecture that addition also needs to be definable in order to have definable Skolem functions.

\section{Subaffine structures}

In this section we study some expansions of the language $\mathcal{L}_{\text {dist }}$ (or rather $\mathcal{L}_{\text {dist }}^{\prime}$ as we should like to achieve quantifier elimination whenever possible). We call these expansions subaffine because we shall only be considering structures $(K, \mathcal{L})$ that are affine in the sense that there does not exist any open subset of $K^{2}$ on which multiplication is $\mathcal{L}$-definable. They are subaffine because addition should not be definable on all of $K^{2}$.

A first, rather trivial example of such an expansion is the language we obtain by adding symbols $\bar{c}$ for the scalar multiplication $\bar{c}: x \mapsto c x$.

$$
\mathcal{L}_{F}:=\{\bar{c}\}_{c \in F} \cup \mathcal{L}_{\text {dist }}^{\prime}
$$

Take fields $F, K$ and $q, q^{\prime} \in \mathbb{N}$ such that $F$ is an $\left(\mathbb{F}_{q^{\prime}}, \mathbb{Z}\right)$-field and $K$ is an $\left(\mathbb{F}_{q}, \mathbb{Z}\right)$-field. We assume that $F \subset K$ and that the valuation on $K$ extends the valuation on $F$ (we shall use the notation ord in both cases). Define the set $\Delta_{K, F}$ to be $\bigcup_{k \in \mathbb{N}} \Delta_{K, F}^{(k)}$, where $\Delta_{K, F}^{(k)}$ is the following set of polynomials

$$
\Delta_{K, F}^{(k)}:=\left\{a x+b y \mid a, b \in F ; x, y \in\left\{x_{1}, \ldots, x_{k}\right\} \cup K\right\}
$$

Let $\bar{\Delta}_{K, F}^{(k)}$ be the set consisting of all constant functions $K^{k} \rightarrow K: x \mapsto a$ and maps $K^{k} \rightarrow K: x \mapsto b x_{i}$, where $a \in K, b \in F$ and $x_{i}$ is one of the variables $x_{1}, \ldots, x_{k}$. Put $\bar{\Delta}_{K, F}:=\bigcup_{k \in \mathbb{N}} \bar{\Delta}_{K, F}^{(k)}$. It is easy to see that the structure $\left(K, \mathcal{L}_{F}\right)$ has cell decomposition and quantifier elimination using $\left(\mathcal{L}_{F}, \Delta_{K, F}, \bar{\Delta}_{K, F}\right)$-cells. The proof is almost literally the same as the corresponding proof for $\mathcal{L}_{\text {dist }}$. Moreover, the scalar multiplication functions we added are the only non-trivial functions, or to be more precise: 
Lemma 4.1 Let $f: A \subseteq K^{k} \rightarrow K^{\ell}$ be a function definable in $\left(K, \mathcal{L}_{F}\right)$. There exists a finite partition of $A$ in $\left(\mathcal{L}_{F}, \Delta_{K, F}, \bar{\Delta}_{K, F}\right)$-cells such that on each cell $C$ the function $f$ has the form

$$
f_{\mid c}: C \rightarrow K^{\ell}: x \mapsto\left(f_{\ell}(x), f_{2}(x), \ldots, f_{l}(x)\right),
$$

where $f_{i}(x)$ is either a constant from $K$ or $f_{i}(x)=a x_{j}$, with $a \in F$ and $x_{j}$ one of the variables $\left\{x_{1}, \ldots, x_{k}\right\}$.

Structures $\left(K, \mathcal{L}_{F}\right)$ do not have definable Skolem functions, as can be seen from the following counterexample:

\section{Lemma 4.2 Let $\Pi$ be the projection map}

$$
\Pi: A:=\left\{(x, y, z) \in K^{3} \mid \operatorname{ord}(z)=\operatorname{ord}(y-x)\right\} \rightarrow K^{2}:(x, y, z) \mapsto(x, y) .
$$

There exists no $\mathcal{L}_{F}$-definable function $f$ such that $\Pi \circ f=\operatorname{Id}_{\operatorname{Im}_{\Pi}}$.

Proof. Suppose that such a function $f$ exists. Up to a finite partition of $\pi(A)$ in cells $\bigcup C_{i} \cup \cup D_{j}$, this function will be of one of the forms

$$
f_{\mid C_{i}}: C \rightarrow K^{3}:(x, y) \mapsto\left(x, y, a_{i}\right) \text { or } f_{\mid D_{j}}: D_{j} \rightarrow K^{3}:(x, y) \mapsto\left(x, y, b_{j} x_{j}\right),
$$

with $a_{i} \in K, b_{j} \in F$ and $x_{j}$ is one of the variables $x$ and $y$.

On cells $C_{i}$, we use a function of the form $(x, y) \mapsto\left(x, y, a_{i}\right)$, which implies that ord $(x-y)=\operatorname{ord}\left(a_{i}\right)$ for all $(x, y) \in C_{i}$. As our partition is finite, we can only have a finite number of cells of this type. Put $M:=$ $\max _{i}$ ord $\left(a_{i}\right)$, then all tuples $(x, y)$ for which ord $(x-y)>M$ will be contained in $\bigcup D_{j}$. For each $k>0$, this set contains elements $(x, y)$ that satisfy

$$
\operatorname{ord}(x)=\operatorname{ord}(y)<M \wedge \operatorname{ord}(x-y)=\operatorname{ord}(x)+k,
$$

which means that we should need a partition in an infinite number of parts $D_{j}$ to define $f$.

This counterexample suggests that it might be impossible to have definable Skolem functions in a language where addition is not definable. This is our main motivation for studying subaffine structures: we shall consider languages $\mathcal{L}_{\boxplus_{0}, K}$ that have a restricted form of addition, and see whether the corresponding structures $\left(K, \mathcal{L}_{\boxplus_{0}, K}\right)$ have definable Skolem functions. Consider the following the functions $\boxplus_{0}$ and $\boxminus_{0}$, defined by

$$
\boxplus_{0}: K^{2} \rightarrow K:(x, y) \mapsto \begin{cases}x+y & x, y \in R_{K} \\ 0 & \text { otherwise }\end{cases}
$$

and analogously for $\boxminus_{0}$, with + replaced by - . Let $K \supset F$ be $\left(\mathbb{F}_{q}, \mathbb{Z}\right)$-fields, resp. $\left(\mathbb{F}_{q^{\prime}}, \mathbb{Z}\right)$-fields. We shall verify that structures $\left(K, \mathcal{L}_{\boxplus_{0}, F}\right)$ have cell decomposition and quantifier elimination, where $\mathcal{L}_{\boxplus_{0}, F}$ is the language

$$
\mathcal{L}_{\boxplus_{0}, F}:=\left\{\boxplus_{0}, \boxminus_{0}\right\} \cup \mathcal{L}_{F} .
$$

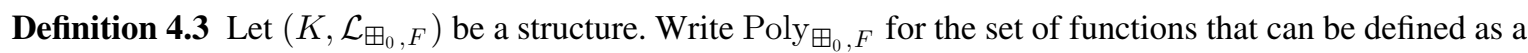
composition of the functions $\boxplus_{0}, \Xi_{0}$ and $\bar{c}$ for $c \in F$, combined with variables $x_{1}, x_{2}, \ldots$ and constants from $K$.

It is important to stress that these expressions do not entirely behave like polynomials. More precisely, we have to be aware that distributivity does not always hold. For example: suppose $0<k_{1}<k_{2}<k_{3}$, then

$$
\pi^{-k_{2}}\left(\pi^{k_{1}} \boxplus_{0} \pi^{k_{3}}\right) \neq \pi^{k_{1}-k_{2}} \boxplus_{0} \pi^{k_{3}-k_{2}}=0 .
$$

First we need to define a notion of cells for this context.

Definition 4.4 Let $\Delta_{\boxplus_{0}, F}$ be the set

$$
\bigcup_{r \in \mathbb{N}}\left\{a\left(x_{1}, \ldots, x_{r}\right)-b\left(x_{1}, \ldots, x_{r}\right) \mid a(x), b(x) \in \text { Poly }_{\boxplus_{0}, F}\right\} .
$$

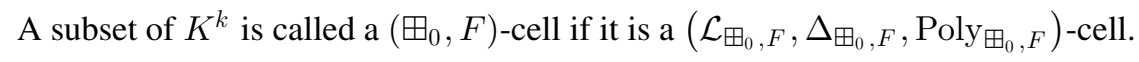


In the next lemmas, we shall show that, up to a finite partition in cells, Poly $\boxplus_{\boxplus_{0}, F}$-functions can always be written in a fairly simple way. Note that for every $\gamma_{0} \in \Gamma_{K}$, the following function is (quantifier free) definable:

$$
\boxplus_{\gamma_{0}}:(x, y) \mapsto \begin{cases}x+y & \text { ord }(x), \text { ord }(y) \geqslant \gamma_{0} \\ 0 & \text { otherwise }\end{cases}
$$

Moreover, we have the following calculation rule. For every $a \in F ; b, c \in K, \gamma \in \Gamma_{K}$ :

$$
a\left(b \boxplus_{\gamma} c\right)=a b \boxplus_{\gamma+\operatorname{ord} a} a c .
$$

Lemma 4.5 Take $\gamma \in \Gamma_{K}, a \in F ; d(x), h(x) \in \operatorname{Poly}_{\boxplus_{0}, F}$. Let $\square$ denote $<, \leqslant,>$ or $\geqslant$. The set

$$
S:=\left\{(x, t) \in K^{k+1} \mid \text { ord }\left(a t \boxplus_{\gamma} d(x)\right) \square \operatorname{ord}(h(x))\right\}
$$

can be partitioned as a finite union of $\left(\boxplus_{0}, F\right)$-cells.

Proof. First note that we may suppose that $a=1$ (if $a t \neq 0$ ), since

$$
\operatorname{ord}\left(a t \boxplus_{\gamma} d(x)\right) \square \operatorname{ord}(h(x)) \Leftrightarrow \text { ord }\left(t \boxplus_{\gamma-\text { ord } a} \frac{d(x)}{a}\right) \square \text { ord }\left(\frac{h(x)}{a}\right) .
$$

Put $a=1$. The set $S$ can then be partitioned as the union of the following three sets:

$$
\begin{aligned}
S= & \left\{(x, t) \in K^{k+1} \mid \operatorname{ord}(t)<\gamma \wedge \operatorname{ord}(0) \square \operatorname{ord}(h(x))\right\} \\
& \cup\left\{(x, t) \in K^{k+1} \mid \operatorname{ord}(t) \geqslant \gamma \wedge \operatorname{ord}(d(x))<\gamma \wedge \operatorname{ord}(0) \square \operatorname{ord}(h(x))\right\} \\
& \cup\left(\left\{(x, t) \in K^{k+1} \mid \operatorname{ord}(t) \geqslant \gamma \wedge \operatorname{ord}(d(x)) \geqslant \gamma\right\}\right. \\
& \left.\cap\left\{(x, t) \in K^{k+1} \mid \operatorname{ord}(t+d(x)) \square \operatorname{ord}(h(x))\right\}\right) .
\end{aligned}
$$

The first two sets are cells. The third set is the intersection of two $\left(\boxplus_{0}, F\right)$-cells and thus again a finite union of cells by Theorem 2.1 .

Lemma 4.6 For $x=\left(x_{1}, \ldots, x_{k}\right)$, and t one variable, let the functions $f_{1}(x, t), \ldots, f_{r}(x, t)$ be in $\mathrm{Poly}_{\boxplus_{0}, F}$. Then $K^{k+1}$ can be partitioned in a finite number of cells $A$, such that on each cell $A$ there are $\gamma_{1}, \ldots, \gamma_{r} \in \Gamma_{K}$, such that either

$$
f_{i}(x, t)=h_{i}(x) \quad \text { or } \quad f_{i}(x, t)=a_{i} t \quad \text { or } \quad f_{i}(x, t)=a_{i} t \boxplus_{\gamma_{i}} d_{i}(x),
$$

with $a_{i} \in F$, and $h_{i}(x), d_{i}(x)$ are in Poly $_{\boxplus_{0}, F}$.

Pro of. We shall work by induction on the number of compositions. Suppose the lemma holds for functions $f$ and $g$. It suffices to check that the lemma also holds for $\bar{c} \circ f$ and $f \boxplus_{0} g$. Take a suitable decomposition into cells $A$. Choose $c \in K$. For all $(x, t) \in A$, the function $\bar{c} \circ f$ will have one of the following forms: either

$$
(\bar{c} \circ f)(x, t)=\bar{c} \circ h_{f}(x) \quad \text { or } \quad(\bar{c} \circ f)(x, t)=c a_{f} t,
$$

or

$$
(\bar{c} \circ f)(x, t)=c\left(a_{f} t \boxplus_{\gamma_{f}} d_{f}(x)\right) .
$$

If we have functions as in (7), we are done. We can rewrite (8) as

$$
c\left(a_{f} t \boxplus_{\gamma_{f}} d_{f}(x)\right)=c a_{f} t \boxplus_{\gamma_{f}+\text { ord } c}\left(\bar{c} \circ d_{f}(x)\right) .
$$

We can apply a similar reasoning to the function $\left(f \boxplus_{0} g\right)$. In most cases, it is obvious that the function has one of the required forms. The only nontrivial cases are when $\left(f \boxplus_{0} g\right)$ has one of the following forms for $(x, t) \in A$ :

$$
\left(f \boxplus_{0} g\right)(x, t)= \begin{cases}a_{f} t \boxplus_{0}\left(a_{g} t \boxplus_{\gamma_{g}} d_{g}(x)\right) & \text { (case 1) } \\ h_{f}(x) \boxplus_{0}\left(a_{g} t \boxplus_{\gamma_{g}} d_{g}(x)\right) & \text { (case 2) } \\ \left(a_{f} t \boxplus_{\gamma_{f}} d_{f}(x)\right) \boxplus_{0}\left(a_{g} t \boxplus_{\gamma_{g}} d_{g}(x)\right) & \text { (case 3) }\end{cases}
$$


Remember that the set $\left\{(x, t) \in K^{k+1} \mid\right.$ ord $\left.\left(a t \boxplus_{\gamma} d(x)\right) \geqslant 0\right\}$ can be written as a finite union of cells, by Lemma 4.5.

We shall check that the lemma holds in case 3 (Case 1 and 2 are similar). Partition $A$ further in cells such that either ord $\left(a_{f} t \boxplus_{\gamma_{f}} d_{f}(x)\right)<0$, or ord $\left(a_{f} t \boxplus_{\gamma_{f}} d_{f}(x)\right) \geqslant 0$ for all $(x, t) \in A$ (and similarly for $g$ ). We only need to consider cells where ord $\left(a_{f} t \boxplus_{\gamma_{f}} d_{f}(x)\right) \geqslant 0$ and ord $\left(a_{g} t \boxplus_{\gamma_{g}} d_{f}(x)\right) \geqslant 0$ as our claim is trivially true on other cells. Partition these cells further depending on the order of $a_{f} t, a_{g} t, d_{f}(x), d_{g}(x)$. The only case that is not immediately obvious is when

$$
\operatorname{ord}\left(a_{f} t\right) \geqslant \gamma_{f}, \text { ord }\left(d_{f}(x)\right) \geqslant \gamma_{f} \text {, ord }\left(a_{g} t\right) \geqslant \gamma_{g} \text { and } \operatorname{ord}\left(d_{g}(x)\right) \geqslant \gamma_{g} .
$$

Let $C$ be such a cell. Without loss of generality, we may suppose that $\gamma_{f} \leqslant \gamma_{g}$. For $(x, t) \in C$ we find that

$$
\begin{aligned}
\left(f \boxplus_{0} g\right)_{\mid C} & =\left(a_{f} t \boxplus_{\gamma_{f}} d_{f}(x)\right) \boxplus_{0}\left(a_{g} t \boxplus_{\gamma_{g}} d_{g}(x)\right) \\
& =\left(a_{f}+a_{g}\right) t+\left(d_{f}(x)+d_{g}(x)\right) \\
& =\left(a_{f}+a_{g}\right) t \boxplus_{\gamma_{f}}\left(d_{f}(x) \boxplus_{\gamma_{f}} d_{g}(x)\right) .
\end{aligned}
$$

Proposition 4.7 Every definable set of $\left(K, \mathcal{L}_{\boxplus_{0}, F}\right)$ can be partitioned as a finite union of $\left(\boxplus_{0}, F\right)$-cells.

Pro of. First we show that quantifier-free definable sets can be partitioned as a finite union of cells. Because of Theorem 2.1, it is sufficient to check that sets of type $S_{1}$ or $S_{2}$ can be partitioned as a finite union of cells:

$$
\begin{aligned}
& S_{1}:=\left\{(x, t) \in K^{k+1} \mid f_{1}(x, t)-f_{2}(x, t) \in \lambda Q_{n, m}\right\}, \\
& S_{2}:=\left\{(x, t) \in K^{k+1} \mid \operatorname{ord}\left(f_{1}(x, t)-f_{2}(x, t)\right)<\operatorname{ord}\left(f_{3}(x, t)-f_{4}(x, t)\right)\right\},
\end{aligned}
$$

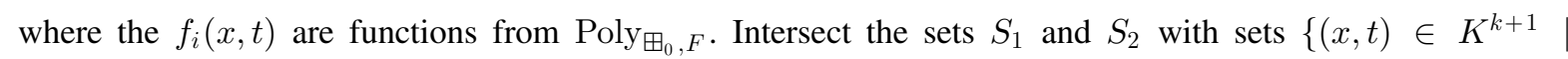
ord $\left(f_{i}(x, t)\right) \square$ ord $\left.\left(f_{j}(x, t)\right)\right\}$ or $\left\{(x, t) \in K^{k+1} \mid \operatorname{ord}\left(f_{i}(x, t)\right) \square \operatorname{ord}\left(h_{j}(x)\right)\right\}$, where $\square$ may denote $<,=$ or $>$. When we apply Theorem 2.1 and Proposition 4.6 to these intersections, it is easy to see that it suffices to check that the sets $\widetilde{S_{1}}$ and $\widetilde{S_{2}}$

$$
\begin{aligned}
& \widetilde{S_{1}}:=\left\{(x, t) \in K^{k+1} \mid a_{1} t \boxplus_{\gamma_{1}} d_{1}(x) \in \lambda Q_{n, m}\right\}, \\
& \widetilde{S_{2}}:=\left\{(x, t) \in K^{k+1} \mid \operatorname{ord}\left(a_{2} t \boxplus_{\gamma_{2}} d_{2}(x)\right)<\operatorname{ord}\left(a_{3} t \boxplus_{\gamma_{3}} d_{3}(x)\right)\right\},
\end{aligned}
$$

can be partitioned as a finite union of cells for all $\gamma_{i} \in \Gamma_{K}$ and $d_{i}(x) \in$ Poly $_{\boxplus_{0}, F}$. For $\widetilde{S_{1}}$ this follows from the observation that the expression $a t \boxplus_{\gamma} d(x) \in \lambda Q_{n, m}$ is equivalent with

$$
\begin{gathered}
\quad[\operatorname{ord}(a t)<\gamma \wedge \lambda=0] \vee[\operatorname{ord}(a t) \geqslant \gamma \wedge \operatorname{ord}(d(x))<\gamma \wedge \lambda=0] \\
\vee\left[\operatorname{ord}(a t) \geqslant \gamma \wedge \operatorname{ord}(d(x)) \geqslant \gamma \wedge\left(t-\frac{-d(x)}{a}\right) \in \frac{\lambda}{a} Q_{n, m}\right] .
\end{gathered}
$$

For the set $\widetilde{S_{2}}$, note that we can restrict our attention to $\widetilde{S_{2}}(1):=\widetilde{S_{2}} \cap A_{\geqslant}$, with

$$
A_{\geqslant}:=\left\{(x, t) \in K^{k+1} \mid \operatorname{ord}\left(a_{i} t\right) \geqslant \gamma_{i} \wedge \text { ord }\left(d_{i}(x)\right) \geqslant \gamma_{i}, \text { for } i \in\{2,3\}\right\},
$$

since it follows easily from Theorem 2.1 that $\widetilde{S_{2}} \backslash{\widetilde{S_{2}}}^{(1)}$ can be partitioned as a finite union of cells. Write $d_{i}^{\prime}(x)=$ $\frac{-d_{i}(x)}{a_{i}}$. Now ${\widetilde{S_{2}}}^{(1)}$ is equal to the set

$$
{\widetilde{S_{2}}}^{(1)}=\left\{(x, t) \in A_{\geqslant} \mid \operatorname{ord}\left(a_{2}\left(t-d_{2}^{\prime}(x)\right)\right)<\operatorname{ord}\left(a_{3}\left(t-d_{3}^{\prime}(x)\right)\right)\right\} .
$$

For $\square=<,=$, or $>$, and put

$$
B_{\square}:=\left\{(x, t) \in K^{k+1} \mid \operatorname{ord}\left(t-d_{2}^{\prime}(x)\right) \square \operatorname{ord}\left(d_{2}^{\prime}(x)-d_{3}^{\prime}(x)\right)\right\} .
$$


Then ${\widetilde{S_{2}}}^{(1)}=\left({\widetilde{S_{2}}}^{(1)} \cap B_{<}\right) \cup\left({\widetilde{S_{2}}}^{(1)} \cap B_{=}\right) \cap\left({\widetilde{S_{2}}}^{(1)} \cap B_{>}\right)$.

Now if ord $\left(t-d_{2}^{\prime}(x)\right)<\operatorname{ord}\left(d_{2}^{\prime}(x)-d_{3}^{\prime}(x)\right)$, then ord $\left(t-d_{3}^{\prime}(x)\right)=\operatorname{ord}\left(t-d_{2}^{\prime}(x)\right)$, so

$$
{\widetilde{S_{2}}}^{(1)} \cap B_{<}=A \geqslant \cap B_{<} \cap\left\{(x, t) \in F^{k+1} \mid \operatorname{ord}\left(a_{2}\right)<\operatorname{ord}\left(a_{3}\right)\right\} .
$$

By Theorem 2.1, this can be written as a finite union of cells. The situation is similar when we intersect with $B_{=}$ or $B_{>}$.

The fact that quantifier-free definable sets can be partitioned as a finite union of cells, also implies that for all $a_{i} \in \Delta_{\boxplus_{0}, F}$, the sets $\left\{x \in K^{k} \mid \operatorname{ord}\left(a_{i}\right) \equiv l \bmod n\right\}$ can be partitioned into $\left(\mathcal{L}_{\boxplus_{0}, F}, \Delta_{\boxplus_{0}, F}\right.$, Poly $\left._{\boxplus_{0}, F}\right)$ precells. Because of this, structures $\left(K, \mathcal{L}_{\boxplus_{0}, F}\right)$ have quantifier elimination by Lemma 2.2 .

The following classification of the definable functions is an immediate consequence of this proposition.

Corollary 4.8 Let $f: A \subseteq K^{\ell} \rightarrow K^{r}$ be an $\mathcal{L}_{\boxplus_{0}, F}$-definable function. There exists a finite partition of $A$ in cells $C$, such that on each cell $C$,

$$
f_{\mid C}(x)=\left(f_{1}(x), \ldots f_{r}(x)\right)
$$

where $f_{i}(x) \in$ Poly $_{\boxplus_{0}, F}$, for $i=1, \ldots, r$.

Proposition 4.9 The addition function $+: K^{2} \rightarrow K:(x, y) \mapsto x+y$ is not definable in $\mathcal{L}_{\boxplus_{0}, F}$.

Pro of. Suppose addition is definable, say by some function $f$. Applying Corollary 4.8 and Lemma 4.6, we can partition $K^{2}$ in cells $C_{i}$ and $D_{i}$ such that

$$
f_{\mid C_{i}}(x, y)=a_{i} x \boxplus_{\gamma_{i, 1}}\left(b_{i} y \boxplus_{\gamma_{i, 2}} c_{i}\right),
$$

and

$$
f_{\mid D_{i}}(x, y)=a_{i} x \quad \text { or } \quad f_{\mid D_{i}}(x, y)=b_{i} y \quad \text { or } \quad f_{\mid D_{i}}(x, y)=c_{i},
$$

for some $a_{i}, b_{i}, \in F, c_{i} \in K$ and $\gamma_{i, 1}, \gamma_{i, 2} \in \Gamma_{K}$. The precise value of these constants depends on $C_{i}$. Put $\gamma:=\min _{i}\left\{\gamma_{i, 1}\right\}$. Clearly all elements $(x, y)$ for which ord $\left(a_{i} x\right)<\gamma$ must be contained in the cells $D_{i}$ since for such elements,

$$
f_{\mid C_{i}}(x, y)=0 \neq x+y .
$$

It is clear that the functions $f_{\mid D_{i}}$ cannot be used to define addition on a large enough set, which proves that the addition function ' + ' is not definable.

The fact that addition is not definable is caused by the fact that we have restricted multiplication to multiplication by a constant. More precisely, the reason is the following (for simplicity, suppose that $\Gamma_{K}$ is $\mathbb{Z}$ ). In our language, it is impossible to take a 'limit' for ord $x$ going to $-\infty$. In a language with normal multiplication, we do not have this restriction, and as a consequence ' + ' can easily be defined in such a language. Take for example the language $\mathcal{L}=\left(\boxplus_{0}, \cdot\right)$. For any $x, y \in K$ with ord $(x) \leqslant$ ord $(y)$ and $x \neq 0$, we can define addition using the following equality.

$$
x+y=x\left(1 \boxplus_{0} \frac{y}{x}\right) .
$$

It is also not hard to see that definable Skolem functions do not always exist for structures $\left(K, \mathcal{L}_{\boxplus_{0}, F}\right)$. Indeed, this follows from the following counterexample.

Lemma 4.10 Let $\Pi$ be the projection map

$$
\left.\Pi:\left\{(x, y, z) \in K^{3} \mid \operatorname{ord}(z)=\operatorname{ord}(y-x)\right\} \rightarrow K^{2}:(x, y, z) \mapsto(x, y)\right\} .
$$

There exists no $\mathcal{L}_{\boxplus_{0}, F}$-definable function $f$ such that $\Pi \circ f=\operatorname{Id}_{\operatorname{Im}_{\Pi}}$. 
Proof. Put $A=\left\{(x, y, z) \in K^{3} \mid\right.$ ord $(z)=$ ord $\left.(y-x)\right\}$. Suppose $f: \Pi(A) \rightarrow A$ is a definable function for which $\Pi \circ f=\operatorname{Id}_{\mathrm{Im}_{\Pi}}$. By Corollary 4.8, there exists a partition of $\Pi(A)$ in cells $C_{i}$ and $D_{i}$ such that

$$
f_{\mid C_{i}}(x, y)=\left(x, y, a_{i} x \boxplus_{\gamma_{i}} h_{i}(y)\right),
$$

and

$$
f_{\mid D_{i}}(x, y)=\left(x, y, a_{i} x\right), \quad \text { or } \quad f_{\mid D_{i}}(x, y)=\left(x, y, h_{i}(y)\right),
$$

with $a_{i} \in F, \gamma_{i} \in \Gamma_{K}$ and $h_{i} \in \operatorname{Poly}_{\boxplus_{0}, F}$. Note that $f_{\mid C_{i}}(x, y)=(x, y, 0)$ for elements $(x, y)$ for which ord $\left(a_{i} x\right)<\gamma_{i}$. So the sets $D_{i}$ must contain all $(x, y)$ for which ord $x$ is too small. However, it is easy to see that the functions $f_{\mid D_{i}}$ do not satisfy our requirements.

This supports our conjecture that structures where addition is not definable do not have definable Skolem functions. Take for example the structure $\left(K, \mathcal{L}_{\boxplus_{0}, K}\right)$. If we fix any constant $\gamma \in \Gamma_{K}$, we can define addition for the set $\left\{(x, y) \in K^{2} \mid \min \{\right.$ ord $(x)$, ord $\left.(y)\} \geqslant \gamma\right\}$. Taking smaller and smaller values for $\gamma$, we can thus define addition on very large open subsets of $K^{2}$, but still not large enough to enable us to define Skolem functions.

While the definability of addition and scalar multiplication seem to be a lower bound for the existence of Skolem functions, it is not a sufficient condition. In a next paper [9] we shall give examples of expansions of $\left(K, \mathcal{L}_{K} \cup\{+\}\right)$ where all bounded semi-algebraic sets are definable, but where definable Skolem functions do not exist.

Acknowledgements The results presented in this paper were obtained as part of my PhD thesis. I would like to thank my supervisor, Raf Cluckers, for many stimulating conversations about this topic, and other members of the jury (in particular, Jan Denef, Angus Macintyre and Leonard Lipshitz) for useful comments. Many thanks also to the Math Department of K.U.Leuven, for providing financial support and a very stimulating working environment. Also thanks to the referee and the editor for pointing out some possible improvements.

\section{References}

[1] Ş. A. Basarab and F. V. Kuhlmann, An isomorphism theorem for Henselian algebraic extensions of valued fields, Manuscr. Math. 77(2-3), 113-126 (1992).

[2] R. Cluckers and E. Leenknegt, A version of $p$-adic minimality, J. Symb. Log. 77(2), 621-630 (2012).

[3] J. Denef, The rationality of the Poincaré series associated to the $p$-adic points on a variety, Invent. Math. 77, 1-23 (1984).

[4] J. Denef, $p$-adic semi-algebraic sets and cell decomposition, J. Reine Angew. Math. 369, 154-166 (1986).

[5] D. Haskell and D. Macpherson, A version of o-minimality for the p-adics, J. Symb. Log. 62(4), 1075-1092 (1997).

[6] F. V. Kuhlmann, Quantifier elimination for Henselian fields relative to additive and multiplicative congruences, Isr. J. Math. 85(1-3), 277-306 (1994).

[7] E. Leenknegt, Cell decomposition for semi-affine structures on $p$-adic fields, J. Log. Anal. 4(14), 1-25 (2012).

[8] E. Leenknegt, Cell decomposition for $p$-adic fields: definable sets and minimality, Ph.D. thesis, K.U. Leuven (2011).

[9] E. Leenknegt, Cell decomposition for semi-bounded $p$-adic sets, Preprint math.LO.1205:4178, submitted (2012).

[10] A. Macintyre, On definable subsets of p-adic fields, J. Symb. Log. 41, 605-610 (1976).

[11] D. Marker, Y. Peterzil, and A. Pillay, Additive reducts of real closed fields, J. Symb. Log. 57(1), 109-117 (1992).

[12] M. H. Mourgues, Cell decomposition for P-minimal fields, Math. Log. Q. 55(5), 487-492 (2009).

[13] Y. Peterzil, A structure theorem for semibounded sets in the reals, J. Symb. Log. 57(3), 779-794 (1992).

[14] Y. Peterzil, Reducts of some structures over the reals, J. Symb. Log. 58(3), 955-966 (1993).

[15] A. Prestel and P. Roquette, Formally $p$-adic fields, Lecture Notes in Mathematics Vol. 1050 (Springer-Verlag, Berlin, 1984).

[16] L. van den Dries, Tame topology and o-minimal structures, London Mathematical Society Lecture Note Series Vol. 248 (Cambridge University Press, Cambridge, 1998). 